\title{
Fundamental Taping Techniques
}

\section{Luka Boban*}

General Hospital Zabok and Croatian Veterans' Hospital, Bračak, Zabok, Republic of Croatia, Balkans

*Corresponding Author: Luka Boban, General Hospital Zabok and Croatian Veterans' Hospital, Bračak, Zabok, Republic of Croatia, Balkans.
Received: February 14, 2020

Published: February 28, 2020

(C) All rights are reserved by Luka Boban.

DOI: 10.31080/ASOR.2020.03.0152

\begin{abstract}
Taping of body parts in sports as means of preventing injury has been a mainstay in high-performance athletes as well as the occasional recreational practitioner. With each sport becoming more demanding and complex as our understanding of the human body and technology improves, the rate of injury increases as well. Taping can be an effective tool in reducing the chances of sustaining injuries, but only if it is applied in the appropriate way. The techniques described are based on anatomic and biomechanic principles and are presented in a systematic format as to ease their memorisation and application. The goal of this article is to demonstrate easy-to-perform taping techniques which can be applied by anyone.
\end{abstract}

Keywords: Taping Techniques; Sport Injuries; Sport Trauma; Injury Prevention

\section{Introduction}

With the advent of organised sports at the begining of the 20th century, the number and popularity of different sport arts has been increasing ever since. Today, technology and facilities allow almost everybody to participate in a sport of some kind all around the world. More sports lead to more athletes which, unfortunately, leads to more injuries. In order to prevent those, athletes and trainers have been searching for the most effective techniques incorporating the newest improvements in technology and medicine as their allies. One of the more prevalent techniques is taping of body parts, taping has been associated with a wide array of positive effects on tissue like joint stabilization, pain reduction and improved joint proprioception [1,2]. When comparing taping to bracing or kinesiotaping, the evidence is often inconclusive and conflicting [3-5]. Literature describing taping techniques is often scarce and most of the techniques are actually passed down wordto-mouth $[3,6]$. This article hopes to provide practical knowledge and skills primarily to individuals involved in sports at all levels of competition; from the occasional „weekend warrior" to highperformance professionals as well as professionals working with and around athletes: kinesiologists, physical therapists, sport physicians, trainers, etc.

The tape used for demonstrative purposes in this article is the $2.5 \times 1400$ centimeters sport tape. While there is a wide array of different-sized tapes avalible on the commercial market, it is encouraged that each practitioner finds the tape that best suits the specific needs of the athlete and activity which is performed and also the one he is most comfortable with working.

Taping techniques

\section{Wrist tape job}

The athlete is positioned in front of the person taping, with the optimal position being a seated one. The hand which we are about to tape is flexed to $90^{\circ}$ in the elbow (Figure 1). The wrist is in neutral position between supination and pronation and the fingers are streched and abducted. 


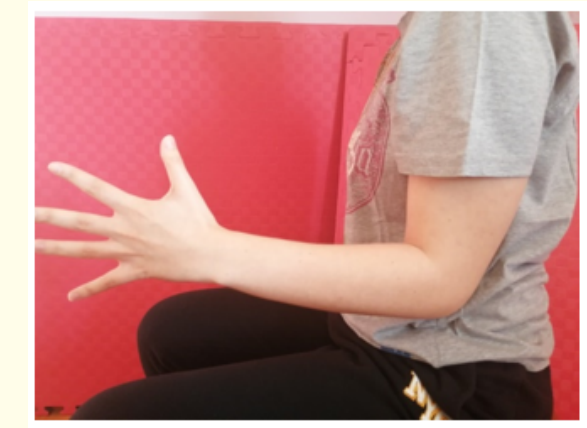

Figure 1: Starting position for the wrist tape job.

The tape job starts with the application of pre-wrap or, as it is more commonly known, „sponge“. The pre-wrap lies under the actual tape and its function is increasing comfort while wearing the tape as well as reducing the number of hairs pulled when removing the tape; its function being purely cosmetic, some practitioners avoid applying pre-wrap because the decreased contact area if the tape with the skin can cause slipping of the tape job.

The pre-wrap is applied from approximately 5 - 7 centimeters proximal from the radiocarpal joint to 2 to 3 centimeters below the heads of the second to fifth metacarpal bones (Figure 2). The wrap is applied in a overlying fashion, with each roll of the wrap covering half of the previous roll. When engaging the thumb, the easiest way is to push through the wrap with the finger while being careful not to damage the pre-wrap too much.

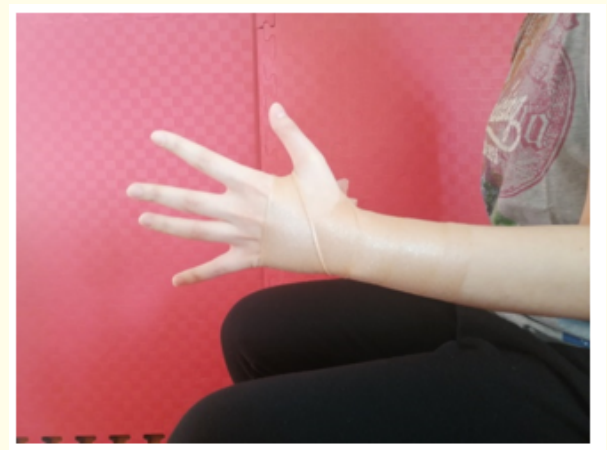

Figure 2: Application of pre-wrap.

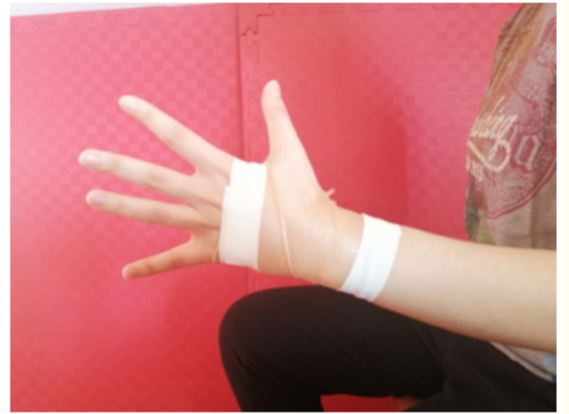

Figure 3: Defining the borders of the tape job.

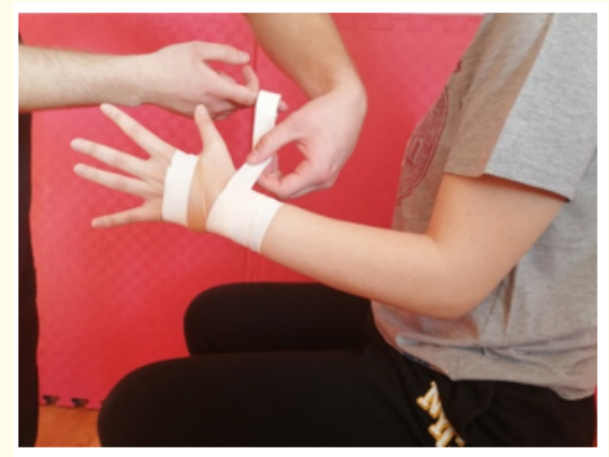

Figure 4: Applying tapestrips in an overlying fashion. 


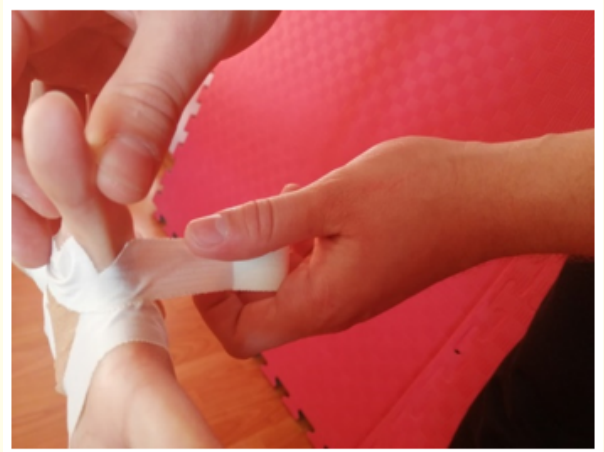

Figure 5: Dividing the tape strip in order to decrease pressure on the web space of the thumb.

With the final tape strip applied over the distal end of the bandage, its "base“ is complete (Figure 6.A. and 6.B). Only small portions of the underlying pre-wrap are seen or none at all. From the base, different approaches possible, defined by the needs of the individual athlete.
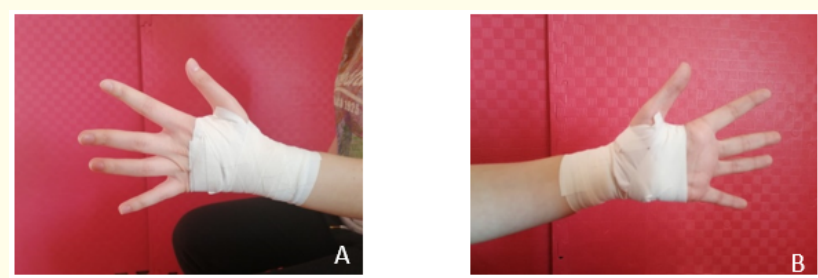

Figure 6: A-B Base of the tape job complete.

The most common technique and the one that will be described here is dorsal enhancement. In order to do so, the hand of the athlete in $10-15^{0}$ of dorsal flexion (Figure 7). A piece of tape is taken and is applied between the borders of the bandage parallel to the axis of the underarm (Figure 8) (it is important that the piece of tape crosses the radiocarpal joint while also taking into account that the strip does not cross the borders of the tape job) (Figure 9). Tension is to be applied on the tape strip while lowering it: the easiest way to do so is to paste the tape distally and then push the thumb into it while simultaneously lowering and pasting the tape (Figure 10). Two more pieces are taken and applied in the exact fashion at an angle of approximately $30-35^{\circ}$ to the first piece, one from the radial and ulnar side respectively (Figure 11). The process can be repeated once or twice more, depending on the needs of the athlete (Figure 12).

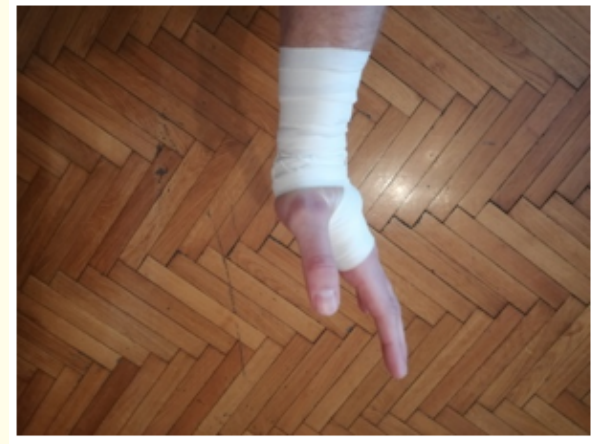

Figure 7: The wrist is positioned in slight dorsal flexion.

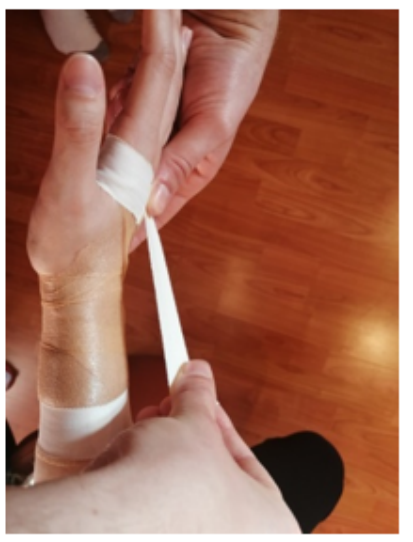

Figure 8: The first strip for dorsal enchancement is placed.

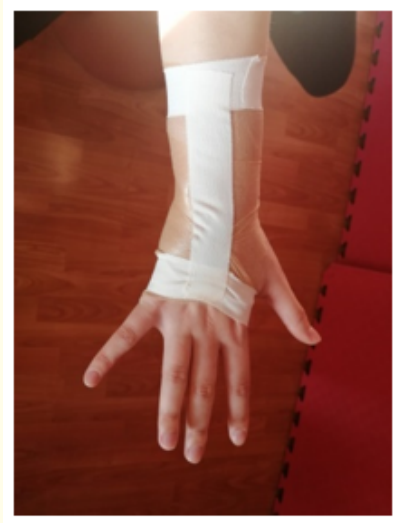

Figure 9: Careful not to cross the borders of the tape job. 


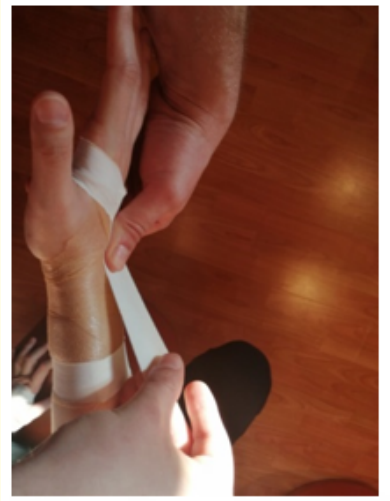

Figure 10: Applying tension to the first dorsal enchancement tape strip.

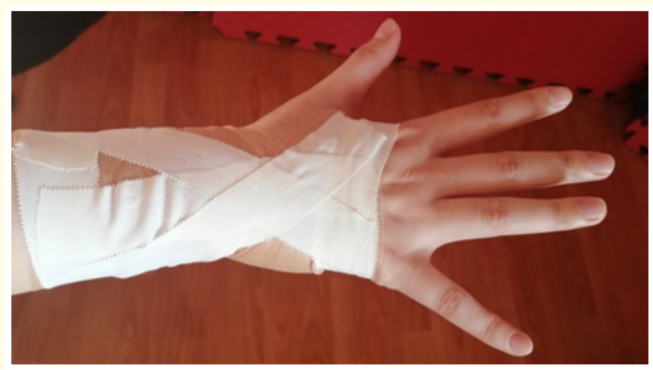

Figure 11: Applying additional tape strips at an angle of 30-35 degrees to the first one.

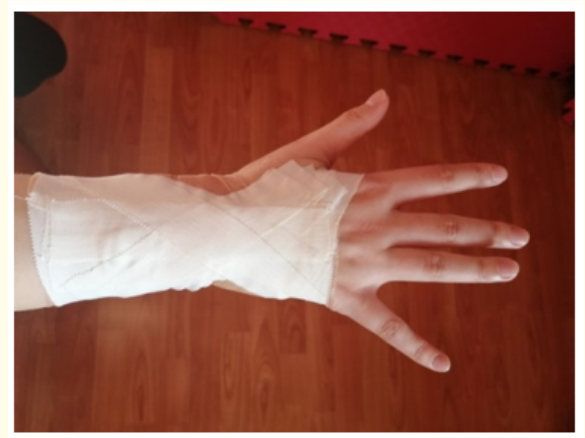

Figure 12: Placing additional tape strips for dorsal enchancement.
Upon completing the dorsal enhancement, the tape job is locked up using locking strips (Figure 13). Once more, the tape is applied in a tile-like fashion from proximal to distal in order to cover loose ends of the tape which could detach following activity or contact with sweat, dirt and other substances.

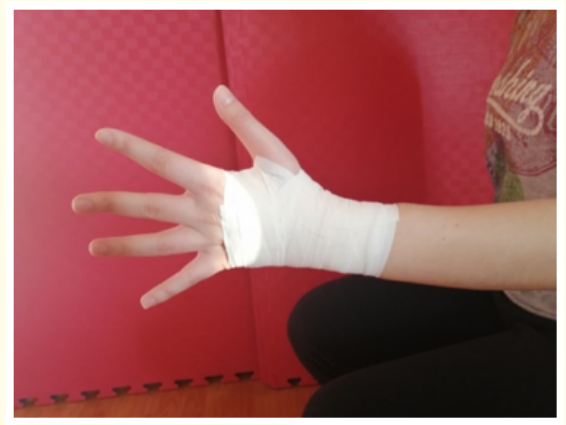

Figure 13: Finishing the tape job with locking strips.

\section{First metacarpal (thumb) joint tape job}

The taping of the thumb joint can be done individually or as an "extension" of the wrist tape job. The recommended technique is the extended one because of its inclusion of the wrist joint: by including the wrist, the hand in total gets added stability as well as preventing the slipping of the tape job due to it lacking a proper „foothold“. For demonstrative purposes, a isolated thumb tape job will be presented.

The athlete is positioned with the underarm in $90^{\circ}$ elbow flexion and the hand in neutral position as seen in Figure 1. The fingers are abducted. The pre-wrap is applied in a similar fashion to the wrist tape job, as is the tape for the base of the tape job.

When the base of the tape job is completed, the position of the hand changes: the fingers are slightly flexed and the hand looks like it is holding a small glass or cup (Figure 14.A. and 14.B.). From the level of the dorsal aspect of the base of the second metacarpal bone, a strip of tape is applied alongside the dorsal aspect of the first metacarpal joint to the palm of the hand (Figure 15.A and 15.B.). This process can be repeated once more if needed, with the second strip lying slightly distal to the first one. 

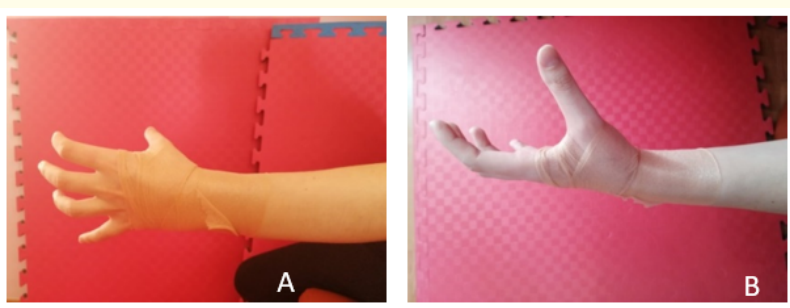

Figure 14: A-B Starting position for the thumb tape job.
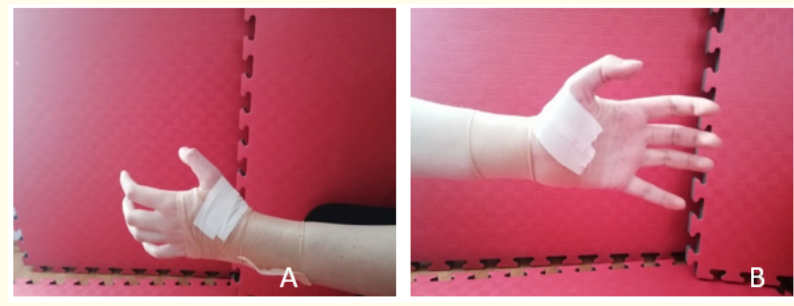

Figure 15: A-B Applying the tape from the dorsal aspect of the second metacarpal bone to the palm of the hand (the borders of the tape job are left out to allow better visualisation).

A strip of tape is then taken and applied from the same point along the ulnar aspect of the thumb joint and back again on the dorsum of the hand, thereby forming a ribbon-like shape (Figure 16.A-C.). The tape can be folded in the region of the interdigital web space as to decrease potential discomfort. Another strip is placed from the thenar of the hand along the ulnar aspect of the joint on the dorsum of the hand and back again on the palmar side, forming the same ribbon-shaped form. The process is repeated once more from each side (Figure 17.A and 17.B.).
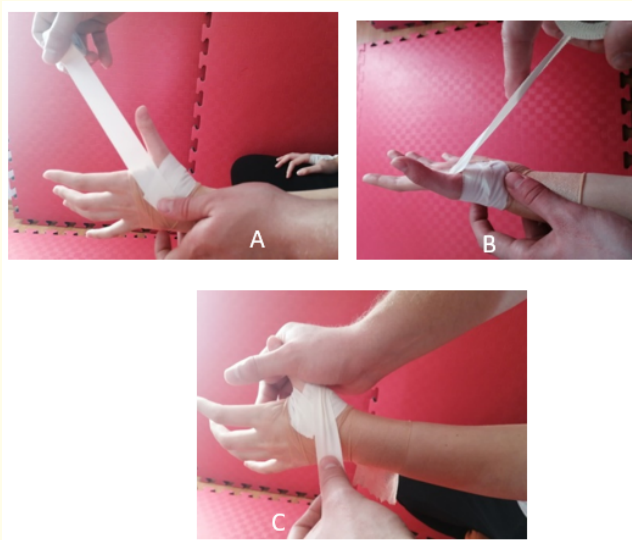

Figure 16: A-C Applying the tape strip around the first metacarpal joint in a ribbon-like fashion starting from the dorso-radial side and finishing dorso-ulnar.
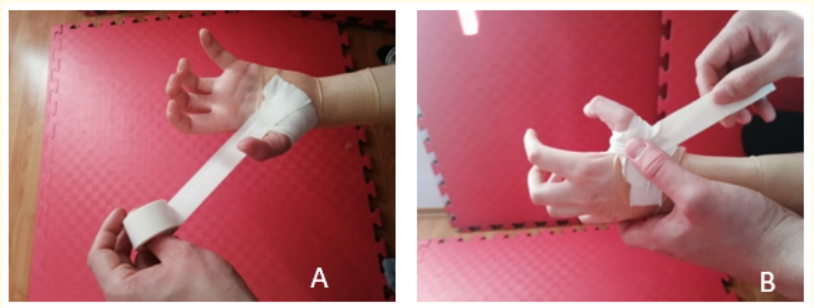

Figure 17: A-B Applying the tape strip around the first metacarpal joint starting from the thenar around the ulnar side of the joint and finishing on the dorsum of the hand forming again a ribbon.

After this step, additional strips are positioned for added support in the same fashion as the ones with which the thumb tape joint was started: from the dorsal aspect of the base of the second metacarpal bone, a strip of tape is applied following the dorsal aspect of the first metacarpal joint to the palm.

After this step, the tape job is locked with locking strips (Figure 18).

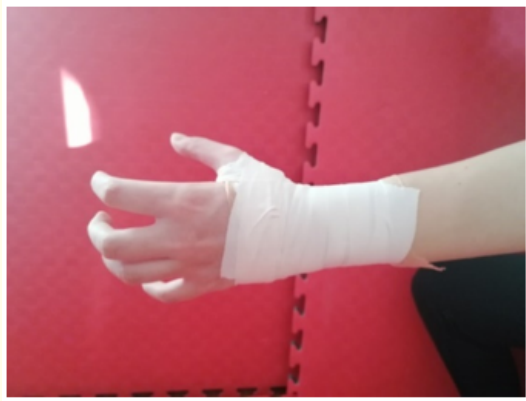

Figure 18: Finishing the tape job with locking strips.

\section{$2^{\text {nd }}-5^{\text {th }}$ finger tape job}

Buddy -tape principle

With the buddy-tape, two fingers are taped together. The problematic or aching finger is taped together with the neighboring healthy one along the proximal and middle phalanx, the metacarpophalangeal joint and the interphalangeal joints being in neutral position. The fingers in question are adducted. Care is to be taken as to not apply the tape over the interphalangeal joints (Figure 19). The number of tape layers applied is defined by athlete demands; the tape should not be applied too tight as not to compromise blood flow through the fingers and induce discomfort. For added cushioning, a piece of pre-wrap can be placed between the fingers prior to taping. 


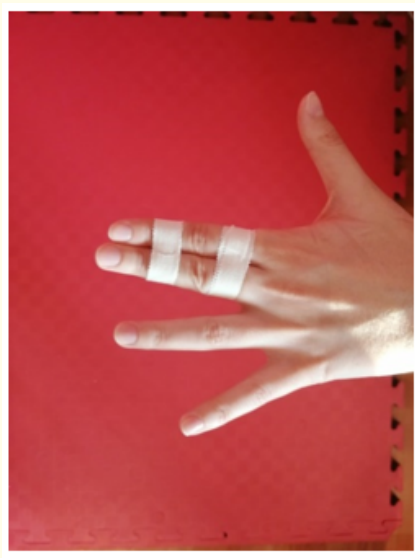

Figure 19: Buddy taping principle, note that the tape strips do not cross the interfalangeal joints.

A variation of the buddy-tape can be made by placing the fingers in a more grip-like position (Figure 20.). This is most often seen in athletes who have needs for griping activities like handball or Brazilian jiu-jitsu. The fingers are displaced from the neutral position and flexed slightly in the metacarpal and interphalangeal joints: the degree of abduction and flexion being defined by the athletes' needs and wishes. The stability of this bandage is questionable when comparing to the standard buddy-tape: if the fingers get adducted, the tape dislodges. To counter this, a piece of pre-wrap can also be applied between the fingers prior to taping, but the padding can get in the way of the potential gripping and holding activities. While it can be beneficial for some athletes, it is not without its shortages.
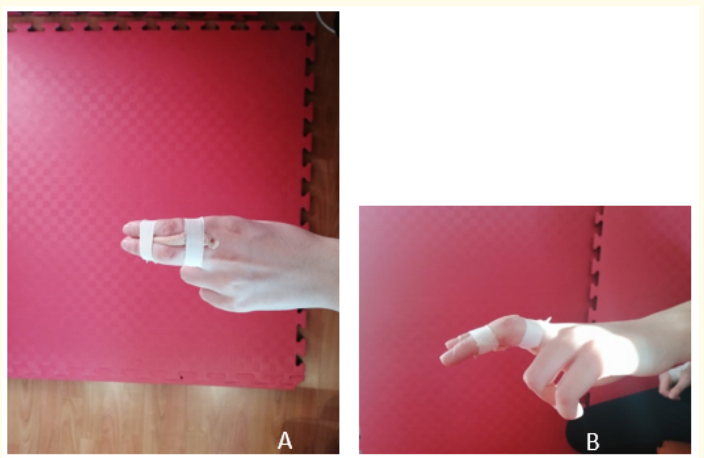

Figure 20: A-B Variation of the buddy taping principle, te prewrap can be seen between the fingers; its purpose being to hold the fingers apart.
Individual tape job of the finger

This taping technique requires no pre-wrap: if the pre-wrap were to be applied, the bandage would easily slip off the finger following sweating.

The finger is flexed slightly in the metacarpal and interphalangeal joints. The tape borders are defined: the proximal end being the middle of the proximal phalanx and the distal end being the distal interphalangeal joint (Figure 21.A and 21.B). Two pieces of tape are applied between the borders over the dorsal aspect of the proximal interphalangeal joint in a oblique fashion: the two pieces should form a "X" shape (Figure 21 and 22).
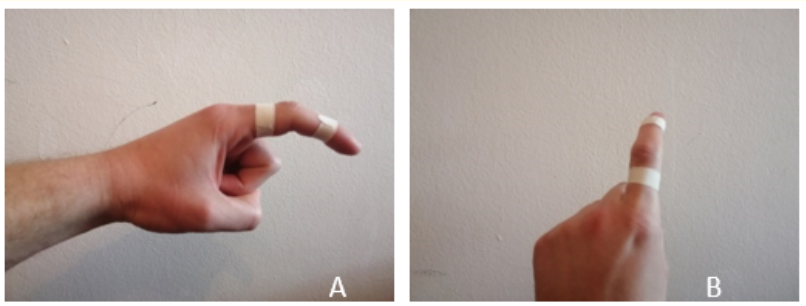

Figure 21: A-B Defining the borders for the individual finger tape job.

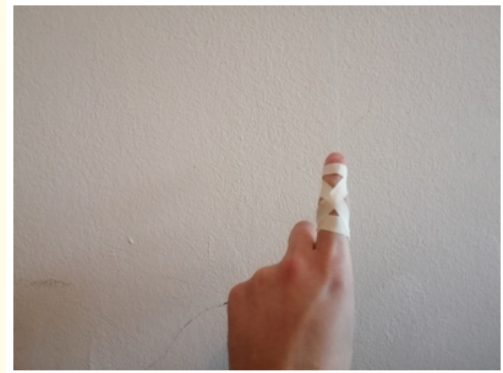

Figure 22: Applying the tape stripes over the proximal interphalangeal joint, thereby forming a "X" shape.

A single piece of tape is taken and placed on the volar side of the finger between the borders of the bandage. This piece is to be applied under tension: first paste the tape along the distal border and the push the tape into the finger with the thumb (Figure 23). Care is to be taken as to maintain the original position of the finger while doing this. 


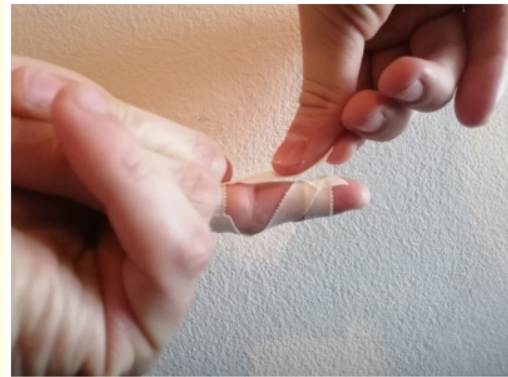

Figure 23: Applying a tape stripe on the volar side of the finger under tension.

The tape is the locked up with locking strips in order to cover the loose ends and „ears" which could potentially loose and lead to tape slippage (Figure 24).

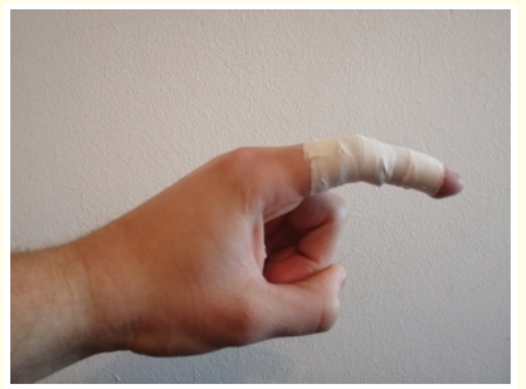

Figure 24: Finishing the tape job with locking stripes.

\section{Ankle tape job}

The most important aspect of the ankle tape job is proper positioning of the athlete: the athlete is best positioned sitting with the knee extended and the leg freely hanging. For added comfort, a cushion can be put under the popliteal fossa and upper aspect of the calf. The foot should be perpendicular to the axis of the lower leg for the duration of the taping (Figure 25).

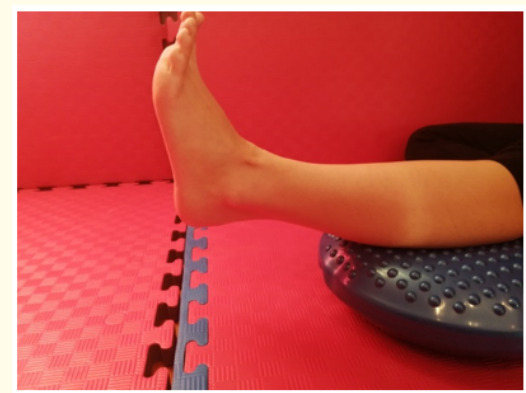

Figure 25: Proper positioning of the foot before begining the tape job.
The pre-wrap is applied from approximately 5 centimeters proximal from the malleoli to just below the metatarsophalangeal joints (Figure 26). The borders of the tape job are defined in the same fashion as in the thumb and wrist jobs: half of the tape strip should be applied directly on the skin, with the other half covering the pre-wrap. The distal border should be angled appropriately to follow the line of the metatarsophalangeal joints (Figure 27.A-B).

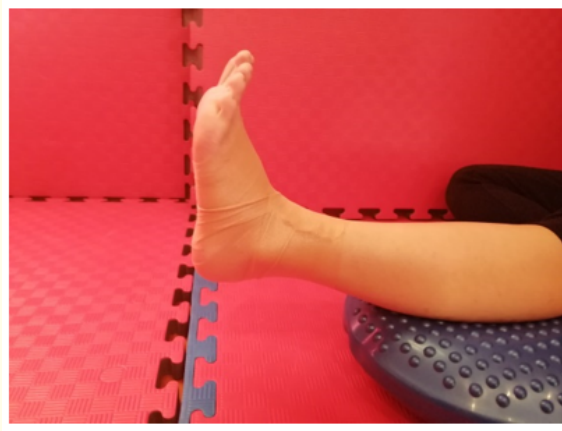

Figure 26: Application of the pre-wrap
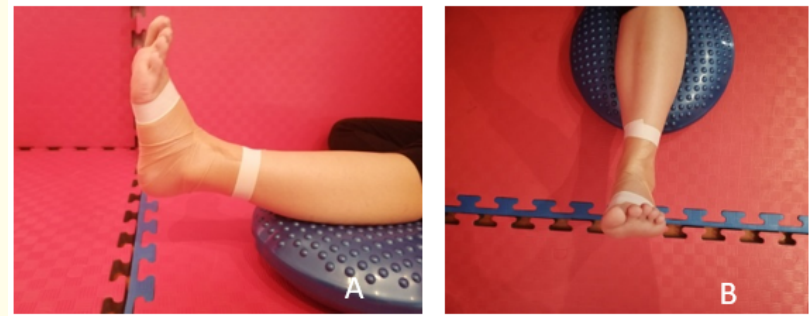

Figure 27: A-B Defining the borders of the tape job.

After defining the borders, the ankle is stabilized in the frontal plane. Three strips of tape are needed, each one the length of the double distance between the thumb and fifth finger of the hand. The first strip is applied from the proximal border parallel to the axis of the lower leg distally, over the underside of the foot and back again to the proximal border forming a "U" shape when looking from the front (Figure 28.A-B). This strip should cover the malleoli. The tape is then molded to follow the physiological contours of the lower leg. The two additional strips are applied in a similar fashion, with one strip lying slightly ventral and the second slightly dorsal to the one overlying the malleoli. The end- product should be a tilelike structure with each strip covering half of the one ventral to itself (Figure 28 and 29).

Additional support is produced by the so-called "figure-ofeights" (for demonstrative purposes, it is shown separately from the rest of the tape job). The starting point is slightly above the line 
connecting the malleoli in the frontal plane and the tape is oriented superolateral. The tape is then applied on the Achilles tendon and then back to above the starting point, completing the first part of the figure-of-eight (Figure 30). The tape is then oriented inferolateral, to the base of the fifth metatarsal bone (Figure 31). Covering the metatarsal, the tape is then taken to the sole of the foot to its medial aspect (Figure 32). Once transcending the medial aspect (Figure 33), the tape is again found on the dorsum of the foot and taken to its origin, completing the second part of the figure-ofeight (Figure 34).
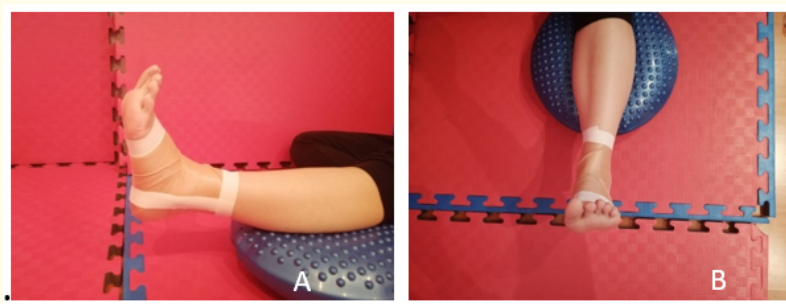

Figure 28: A-B Stabilizing the ankle joint in the frontal plane, the first tape strip should cover both maleoli.

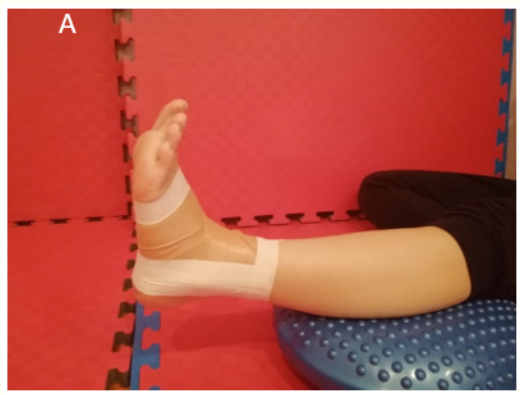

Figure 29: Two additional tape strips are applied, overlying the first one.

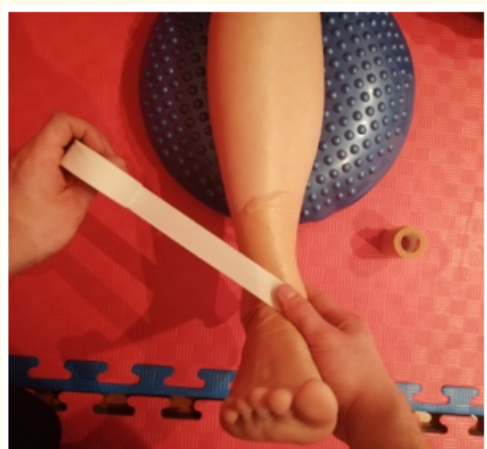

Figure 30: Starting position for the figure-of-eight.

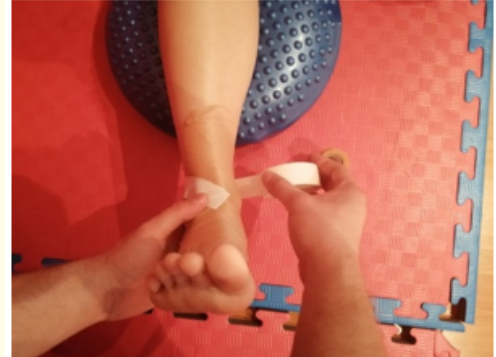

Figure 31: Comming behind the Achilles tendon.

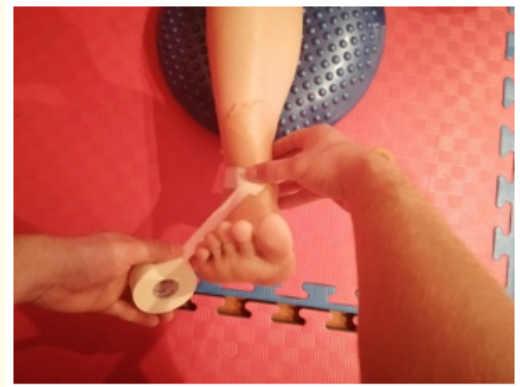

Figure 32: Continuing the figure-of-eight, the tape is oriented toward the base of the fifth metatarsal bone.

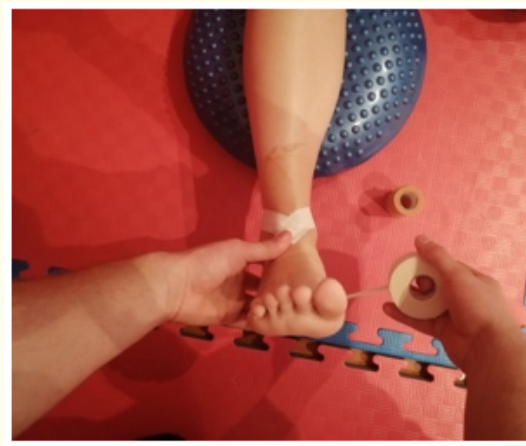

Figure 33: Approaching the medial border of the foot sole.

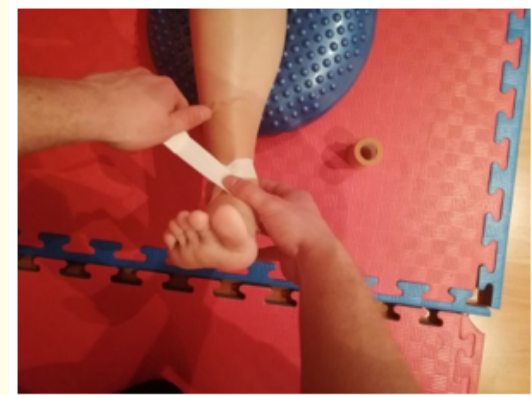

Figure 34: Finishing the figure-of-eight. 
For added stability and safety, a second figure-of-eight is applied, the application of which is a mirror image to the previous one. The tape is oriented superomedial and dorsally behind the Achilles tendon and back to the starting point; the tape is then oriented to the medial aspect of the sole. Once covering the sole, the tape "emerges" above the fifth metatarsal and is oriented to its origin (Figure 36.A-E).

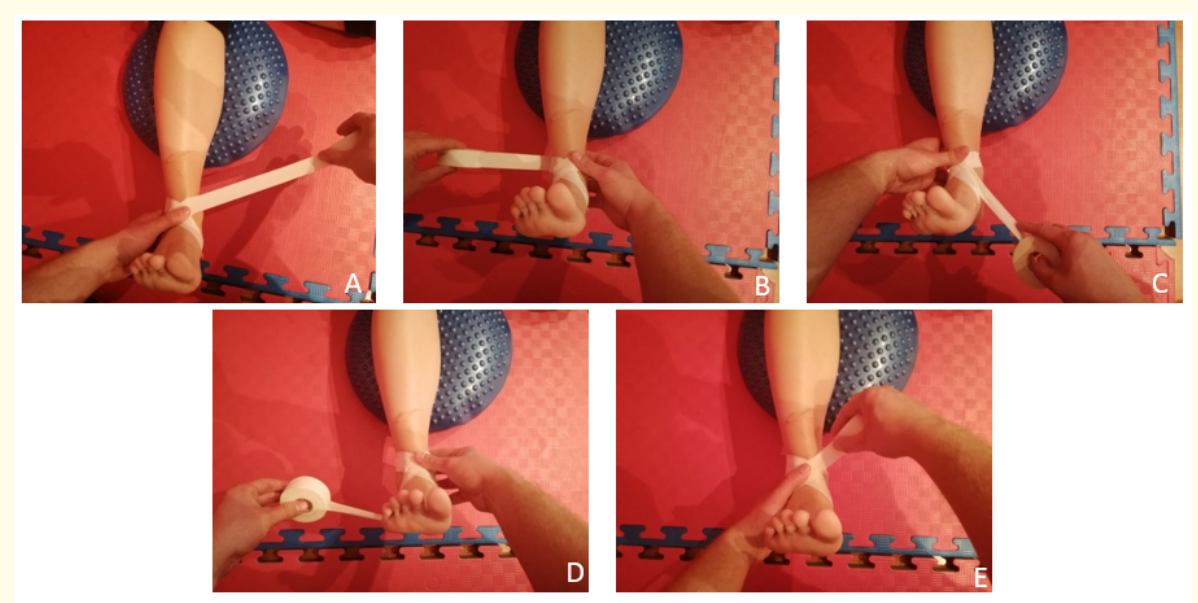

Figure 35: A-E Applying the second figure-of-eight, mirroring the one previously described.

Once the figure-of-eights are completed, the ankle job can be extended by a heel-lock (see under Heel-lock) or it can be locked with standard locking strips (Figure 36).
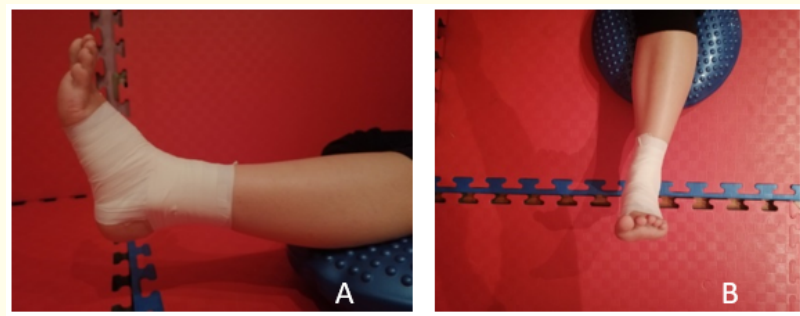

Figure 36: A-B Finishing the ankle tape job with locking strips.

\section{Heel lock}

A heel lock can be part of the standard ankle tape job or an individual taping technique. It is recommended for athletes who have complaints regarding their calcaneus or general ankle stability. The heel lock in itself is a modification of the previously described figure-of-eight. For demonstrative purposes, an isolated heel-lock will be formed but the recommendation is to include it in the standard ankle tape job.

Starting from the medial malleolus, the tape is oriented over the dorsum of the foot to the fifth metatarsal (Figure 37). When approaching the sole of the foot, the tape should be oriented slightly towards the calcaneus. When approaching the medial aspect of the foot, the tape is oriented parallel to the calcaneus and "hugs" it alongside its posterior processus (Figure 38). Approaching the lateral malleolus, the tape is oriented towards it point of origin, thereby completing the first heel-lock (Figure 39 and 40). Like with figure-of-eights, the second heel-lock is a mirror image of the first one: starting from the lateral malleolus, the tape strip is oriented over the dorsum of the foot to the medial aspect of the sole. From the underside of the foot the tape is oriented slightly towards the calcaneus and "hugs" it in the same fashion as described previously. When approaching the medial malleolus, the tape is brought back to its origin (Figure 40.A-E). The tape job is locked with locking strips as shown in Figure 37.

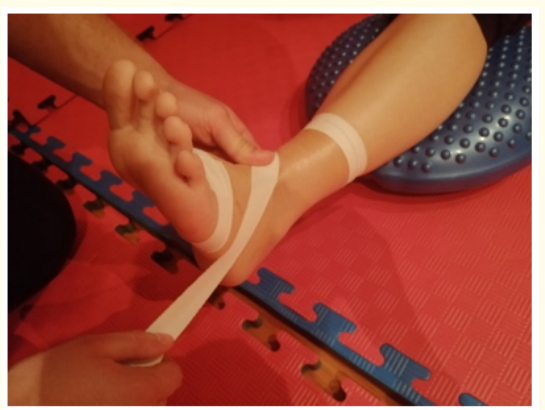

Figure 37: Starting position for theheel lock. 


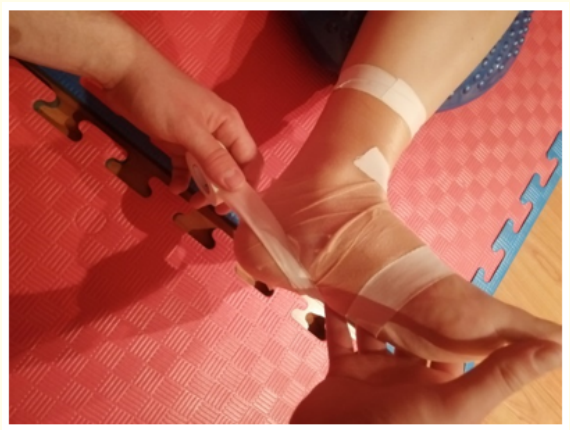

Figure 38: Coming from the underside of the foot and "hugging" the calcaneus.

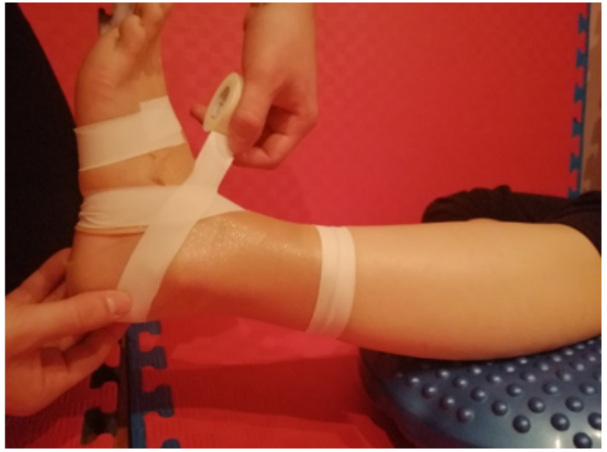

Figure 39: Finishing the first heel lock.
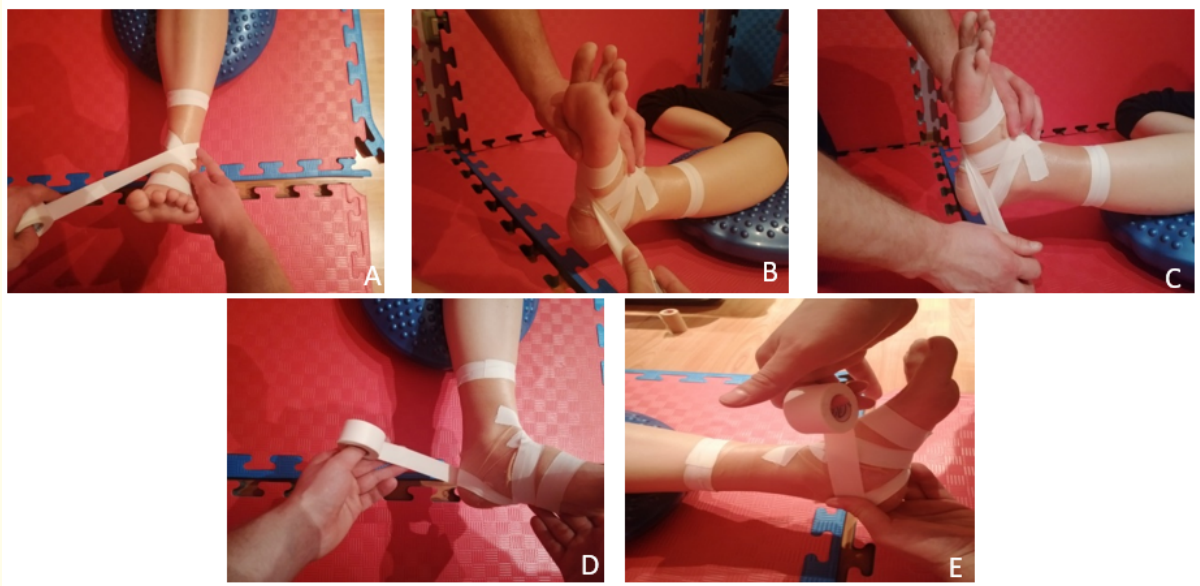

Figure 40: A-E Applying the second heel lock.

\section{Toe tape job}

Taping technique for toes all follow a similar principle. A strip of tape is taken and applied from the underside of the metatarsophalangeal joint over its dorsal aspect in a ribbon-like form. This process can be repeated once or twice more, depending on the demands of the athlete (Figure 41). Note that comfort is of exceptional importance in this area, as wrongly applied tape can lead to skin excoriations, blisters and pain. Because of its size, the big toe can be addittionaly stabilized by applying a strip of tape over its dorsal and plantar sides (Figure 42). Additional tape stripes are applied from the underside of the toe over the metatarsophalangeal joint (Figure 43) It is recommended to combine this technique with the standard ankle tape job as to avoid slippage of the great toe tape. The tape can then be locked into place with standard locking strips (Figure 44.A-B).

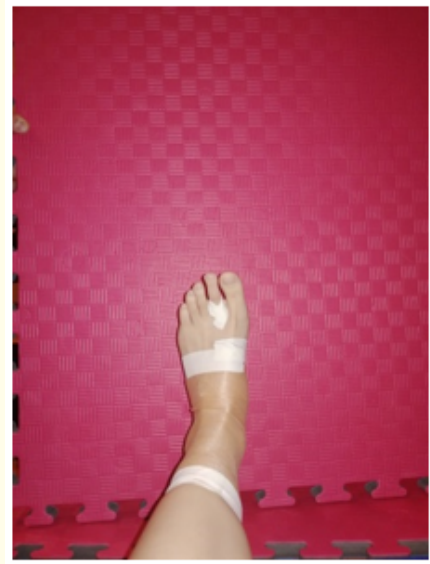

Figure 41: Tape job for toes. 


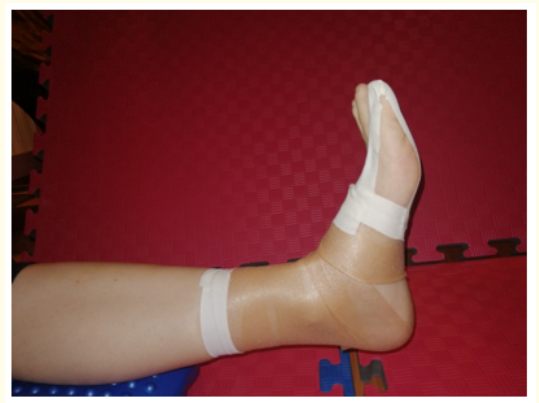

Figure 42: Application of the tape strip over the big toe, note that the strip is "anchored" at the distal border of the tape job.

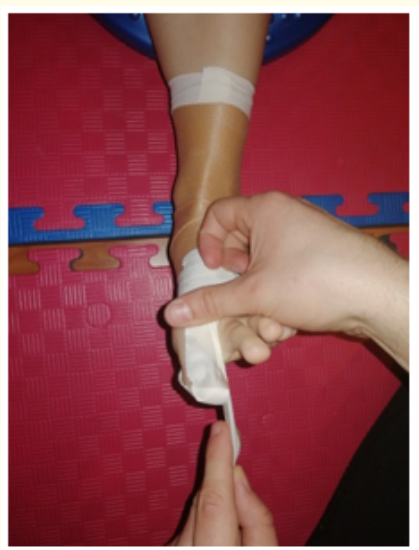

Figure 43: Application of tape strips from the underside of the metatarsophalangeal joint in a ribbon-like fashion.
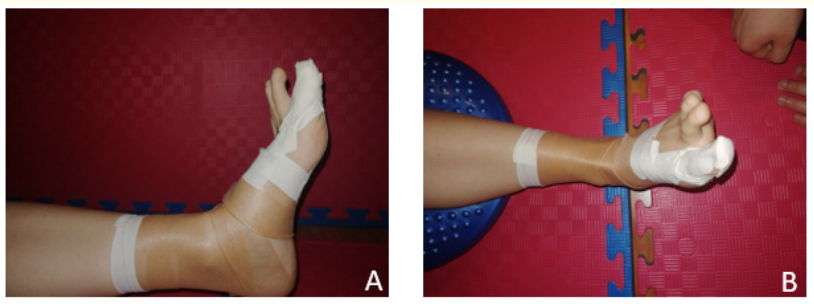

Figure 44: A-B Finishing the tape job with locking strips.

\section{Conclusion}

While most of the literature available on the same subject focuses on the biomechanical effect of taping on tissues, this article hopes to give its readers practical knowledge essential to properly apply the taping techniques described. As our understanding of the human body grows and improves, new and effective ways of prevention will most definitely be defined. Nonetheless, taping, as a simple, cheap, and globally available preventive method, will always have its place in sport medicine.

\section{Bibliography}

1. Bahr R and Bahr IA. "Incidence of acute volleyball injuries: a prospective cohort study of injury mechanisms and risk factors". Scandinavian Journal of Medicine and Science in Sports 7.3 (1997): 166-171.

2. Halim-Kertanegara S., et al. "The effect of ankle taping on functional performance in participants with functional ankle instability". Physical Therapy in Sport 2 (2017): 162-167.

3. Trégouët P., et al. "A comparison of the effects of ankle taping styles on biomechanics during ankle inversion". Annals of Physical and Rehabilitation Medicine 56.2 (2013): 113-122.

4. Nunes GS., et al. "Kinesio Taping does not decrease swelling in acute, lateral ankle sprain of athletes: A randomised trial". Journal of Physiotherapy 61.1 (2015): 28-33.

5. Lee SM and Lee JH. "The immediate effects of ankle balance taping with kinesiology tape on ankle active range of motion and performance in the Balance Error Scoring System". Physical Therapy in Sport 25 (2017): 99-105.

6. Porretto-Loehrke A. "Taping techniques for the wrist". Journal of Hand Therapy 29.2 (2016): 213-216.

\section{Assets from publication with us}

- Prompt Acknowledgement after receiving the article

- Thorough Double blinded peer review

- Rapid Publication

- Issue of Publication Certificate

- High visibility of your Published work

Website: https://www.actascientific.com/

Submit Article: https://www.actascientific.com/submission.php Email us: editor@actascientific.com

Contact us: +919182824667 